Original

\title{
Vascular Lesion in the Meninx and Retina Following Administration of 3,3'- Iminodipropionitrile (IDPN) in Rats
}

\author{
Kotaro Yamashita ${ }^{1}$, Yoshimasa Okazaki $^{1, \#}$, and Minoru Tsuchitani ${ }^{1}$ \\ ${ }^{1}$ Mitsubishi Chemical Safety Institute Ltd., 14 Sunayama, Hasaki, Kashima, Ibaraki 314-0255, Japan \\ \# Present: Department of Toxicologic Pathology, Toxicology Research Laboratories, Fujisawa Pharmaceutical Co., Ltd., \\ Kashima 2-1-6, Yodogawa, Osaka 532-8514, Japan
}

\begin{abstract}
Iminodipropionitrile (IDPN) is a potent neurotoxicant, which produces behavioral abnormalities and neuropathologic lesions in the central and peripheral nervous systems. Recently, 11 laboratories including ours carried out a collaborative neurotoxicity study using IDPN, and arterial hyalinization in the meninx of the brain and spinal cord was detected in 6 laboratories. Before that study, IDPN-induced vascular lesion had not been reported in any organ except the retina. The present study was undertaken to confirm the reproducibility of the IDPN-induced vascular lesion, to find out the onset of the lesion, and to determine the systemic distribution of the lesion. Male Crj:CD(SD)IGS rats were treated daily with IDPN $125 \mathrm{mg} / \mathrm{kg}$ and were sacrificed on days 15,30 , and 44 . Behavioral abnormalities appeared from day 9 and thereafter, and axonal swelling in the central nervous system (CNS) was found on days 15, 30, and 44. Arterial hyalinization in the meninx was seen in 4 and 5 out of 6 rats on days 30 and 44, respectively. Almost all lesions were found in the small sized arteries in the basilar area of the brain and spinal cord, and near the dorsal roots of the spinal cord. Some affected vessels demonstrated fibrinoid degeneration, inflammatory cell infiltration or occluded lumina due to intimal thickening. In the organs and tissues other than the CNS, arterial hyalinization in the retina was found on days 30 and 44. Our results demonstrate the reproducibility of IDPN-induced vascular lesions and suggest that the lesions characterized by arterial hyalinization are restricted to the CNS and retina, and that vascular lesion occurred following neurotoxicity. (J Toxicol Pathol 2005; 18: 19-25)
\end{abstract}

Key words: 3,3'-iminodipropionitrile (IDPN), arterial hyalinization, meninx, retina, rat

\section{Introduction}

3,3'-Iminodipropionitrile (IDPN), a well-known industrial intermediate, is a potent neurotoxicant that induces a characteristic neurobehavioral syndrome consisting of repetitive head movement, retropulsion, circling, and hyperactivity ${ }^{1,2}$. Moreover, rats exposed to IDPN show proximal axonal swelling with accumulated neurofilaments in the central and peripheral nervous system ${ }^{3-5}$. Other adverse effects of IDPN in the rat include olfactory toxicity resulting from degeneration of the olfactory mucosa ${ }^{6}$, ototoxicity resulting from the loss of hair cells in the organ of Corti $^{7}$, vestibular toxicity resulting from degeneration of the vestibular sensory hair cells ${ }^{8}$, and visual toxicity resulting from retinal degeneration?.

A collaborative neurotoxicity study using IDPN with a common protocol based on the Organization for Economic

Received: 22 October 2004, Accepted: 31 January 2005 Mailing address: Kotaro Yamashita, Mitsubishi Chemical Safety Institute Ltd., 14 Sunayama, Hasaki, Kashima, Ibaraki 314-0255, Japan

TEL: 81-479-46-2871 FAX: 81-479-46-2874

E-mail: k-yamashita@ankaken.co.jp
Cooperation and Development (OECD) test guideline TG407 was conducted by 11 laboratories in Japan ${ }^{10}$. In the histopathological examination of this study, which was restricted to the central and peripheral nervous systems, we identified angitis or hyalinization of the vascular wall in the meninx of rats receiving $100 \mathrm{mg} / \mathrm{kg}$ of IDPN for 28 days. The vascular lesions were confirmed by 6 laboratories, including ours, however, the lesions were not found in rats at the other 5 laboratories.

Heath et al. ${ }^{11,12}$ demonstrated that administration of IDPN caused retinal angiopathy, characterized by endothelial proliferation and deposition of Periodic acidSchiff (PAS)-positive material in the arterial wall, however, the cause of the retinal angiopathy is not known.

There is no report concerning the vascular toxicity of IDPN in organs other than the retina and the central nervous system (CNS). The present study, therefore, was undertaken to confirm the reproducibility of the IDPN-induced vascular toxicity in the retina and meninx, and to find out the onset of the lesion. Additionally, a complete histopathologic examination was performed to identify the distribution of the vascular lesions due to IDPN intoxication in rats. 


\section{Materials and Methods}

\section{Chemicals}

3,3'-Iminodipropionitrile (IDPN, CAS No. 111-94-4, molecular wt. 123.16, 98\% pure) was obtained from Wako Pure Chemicals, Industries Ltd. (Kyoto, Japan) and dissolved in purified water at a concentration of $25 \mathrm{mg} / \mathrm{ml}$ for the dosing solution.

\section{Animals}

Sixty-two male Crj:CD(SD)IGS rats were obtained from Charles River Japan (Kanagawa, Japan). The animals were individually housed in wire-mesh cages and the room was automatically controlled at a temperature of $20-24^{\circ} \mathrm{C}$ with a relative humidity of $40 \%-70 \%$ under a 12 -h light/dark cycle. The rats were given a commercially available diet (MF, Oriental Yeast Co., Ltd, Tokyo, Japan) and tap water ad libitum. Healthy rats were used for the study after an acclimatization period of 5 days.

The animals were maintained according to the principles outlined in the guide for the care and use of laboratory animals prepared by the Japanese Association for Laboratory Animal Science and our institution.

\section{Treatment and clinical observations}

Sixty rats were stratified by body weight, and were divided into two experimental groups to create similar initial body weights. Groups of 30 rats at 7 weeks old were treated orally with $125 \mathrm{mg} / \mathrm{kg}$ per day of IDPN or purified water (control group). The dosage level of IDPN was established according to the results of the previously mentioned collaborative neurotoxicity study. In the collaborative study, vascular lesions in the brain and spinal cord were seen in 6 of 11 laboratories, however, there were no dead or moribund rats at treatments of $100 \mathrm{mg} / \mathrm{kg}$ IDPN per day ${ }^{10}$. Therefore, a dosage slightly higher than that of the collaborative study was selected for the present study in order to be more certain of inducing the vascular lesions.

All rats were carefully observed for clinical signs once a day. Body weights were recorded before dosing and on days 3 and 8 , and once a week thereafter.

\section{Histopathologic examinations}

Groups of IDPN-treated rats $(\mathrm{n}=6)$ and control rats $(\mathrm{n}=6)$ were sacrificed in numerical order on days 15,30 , and 44 , by exsanguination from the abdominal aorta under sodium thiopental anesthesia. Histopathologic examination was conducted using 39 rats including three rats that were euthanatized due to their moribund condition. The other 21 rats were used in another study investigating nerve conduction velocity.

The following organs and tissues were collected and fixed in $10 \%$ neutral buffered formalin solution by immersion: adrenals, aorta at the thoracic level, bones with bone marrow including femur bone and sternum bone, brain, epididymides, esophagus, eyeballs and optic nerves, Harderian glands, heart, kidneys, large intestines including cecum, colon, and rectum, lungs, liver, lymph nodes including mandibular lymph nodes and mesenteric lymph nodes, mammary glands, pancreas, parathyroids, pituitary, prostate, salivary glands including sublingual glands and submandibular glands, sciatic nerves, seminal vesicles, skeletal muscle, skin, small intestines including duodenum, jejunum, and ileum, spleen, spinal cord, stomach, testes, thymus, thyroids, tongue, trachea, and urinary bladder. The brain was cut coronally through the frontal lobe, forward infundibulum, and culmen of the cerebellum. The spinal cord was cut transversely at the cervical, thoracic, and lumbar levels. Tissue samples were processed for paraffin embedding, sectioned at $5 \mu \mathrm{m}$ in thickness, and stained with hematoxylin and eosin (HE). Some selected sections obtained from the brain, spinal cord, and eyeballs, were stained with periodic acid Schiff (PAS), phosphotungstic acid hematoxylin (PTAH) and elastica van Gieson (EVG). All preparations were then examined by a light microscope.

\section{Electron microscopical examinations}

Small pieces of brain tissues with the meninx of one IDPN-treated rat and one control rat originally fixed in formalin were post-fixed in $1 \%$ osmium tetroxide in phosphate buffer, and then dehydrated and embedded in epoxy resin in accordance with routine methods. Ultrathin sections were double-stained with uranyl acetate and lead citrate, and examined under an electron microscope (JEM100CX II, JEOL, Tokyo, Japan).

\section{Results}

\section{Mortality, clinical signs and body weight changes}

IDPN-induced typical symptoms such as circling, head bobbing, and retropulsion which appeared from day 9 and thereafter. Abnormal gait was seen from day 10 and thereafter. These changes were observed among no less than 25 out of 30 rats treated with IDPN. After day 38, wryneck, mydriasis, and incontinence of urine were also noted in IDPN-treated rats. One rat was found dead on day 38 and 3 rats were euthanatized due to deterioration of their general condition on days 39 or 42 .

Body weights of IDPN-treated rats were almost constant throughout the dosing period (Fig. 1). Body weights of half of the rats on day 28 and almost all rats on day 43 were lower than those on day 1.

\section{Vascular lesions}

Vascular lesions were found in the brain, spinal cord, and retina. The incidence of the lesions is summarized in Table 1.

Arterial hyalinization in the meninx of the brain was found in five out of six rats that were sacrificed on each of days 30 and 44 . Arterial hyalinization in the meninx of the spinal cord was found in four out of six rats that were sacrificed on day 30 , and five out of six rats that were sacrificed on day 44. Most of the affected vessels had thickened walls, due to deposition of PAS-positive and 
PTAH-negative materials (Figs. 2a, 2b, and 2c). Rupture of the internal elastic lamina (IEL) was occasionally observed in the affected vessels (Fig. 2d). Some of the affected vessels showed fibrinoid degeneration (Figs. 2e and 2f), endothelial proliferation with inflammatory cell infiltration (Fig. 2g), or were occluded due to the thickened intima (Fig. $2 \mathrm{~h})$. Such lesions in the brain and spinal cord were also found in all the three rats that were euthanatized due to their moribund condition. No vascular lesion was found in the parenchyma of the brain and spinal cord.

The distribution of the vascular lesions in the meninges of the brain and spinal cord is shown in Fig. 3. The vascular lesions were distributed among small sized arteries in the basilar area of the brain and spinal cord, and near the dorsal

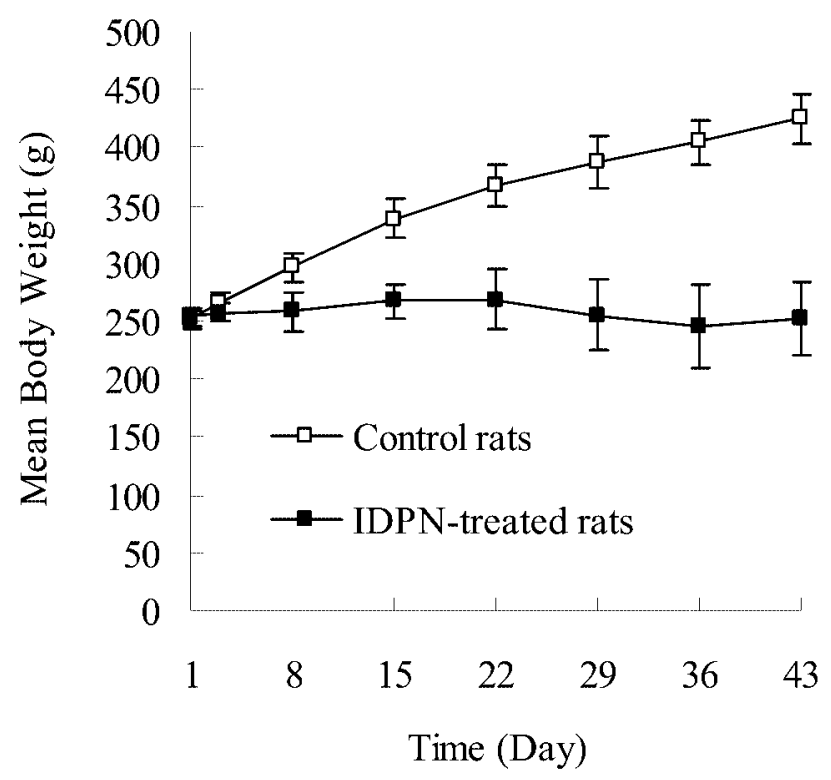

Fig. 1. Mean body weights of rats receiving $125 \mathrm{mg} / \mathrm{kg}$ per day of IDPN or purified water (control). Error bars show the standard deviation. roots of the spinal cord. In some cases, vascular lesions were also found on the upper side of the paraflocculus of the cerebellum.

Arterial hyalinization was also present in the retina of the eyes (Fig. 4d). The lesion was found in all IDPN-treated rats that were sacrificed on days 30 and 44, and two rats that were euthanatized due to their moribund condition (Table 1). Most of the affected vessels had thickened walls due to deposition of PAS-positive materials (Fig. 4e), and they also had incomplete IEL (Fig. 4f). The lesions were accompanied by retinal edema. Such changes were not found in the retina of the eyes from rats of the control group (Fig. $4 \mathrm{a}-\mathrm{c}$ ). Most of the affected vessels were located near the optic disc. In severe cases, however, lesions were present throughout the retina. Keratitis and iritis occurred sporadically in the eyes, with or without vascular lesions, of rats treated with IDPN.

No vascular lesion was found in any other organ or tissues examined in this study.

\section{Neuropathologic changes}

Axonal swelling, the neuropathologic hallmark of IDPN intoxication, in the brain and spinal cord or in the peripheral nerves was found in all rats that were sacrificed on days 15,30 , and 44 , and those that were euthanatized due to their moribund condition.

Axonal swelling was seen frequently in the reticular nucleus, spinal trigeminal tract, and vestibular nucleus of the pons, as well as in the ventral horn and ventral funiculus of the spinal cord. The lesions were also seen in the dorsal horn of the spinal cord (Fig. 3).

\section{Electron microscopic finding}

The lumen of the affected pial artery of the brain was occupied by proliferating endothelial cells. Electron dense amorphous material was deposited in the subendothelial space near the internal elastic lamina. Thinning of internal elastic lamina was also observed (Fig. 5).

Table 1. Incidences of Arterial Lesions Induced by Repeated Doses of IDPN*

\begin{tabular}{|c|c|c|c|c|c|c|}
\hline \multirow{3}{*}{$\begin{array}{l}\text { Organs and tissues } \\
\text { Findings }\end{array}$} & \multirow{3}{*}{$\begin{array}{l}\text { Experimental day of necropsy } \\
\text { Number of rats examined }\end{array}$} & \multicolumn{3}{|c|}{ Scheduled sacrifice } & \multicolumn{2}{|c|}{ Moribund sacrifice } \\
\hline & & 15 & 30 & 44 & 39 & 42 \\
\hline & & 6 & 6 & 6 & 1 & 2 \\
\hline \multicolumn{7}{|l|}{ Meninx of the brain } \\
\hline Hyalinization & & 0 & 5 & 5 & 1 & 2 \\
\hline Fibrinoid degene & & 0 & 2 & 0 & 1 & 2 \\
\hline Inflammatory ce & ltration & 0 & 1 & 0 & 0 & 0 \\
\hline Endothelial proli & & 0 & 1 & 0 & 0 & 0 \\
\hline \multicolumn{7}{|c|}{ Meninx of the spinal cord } \\
\hline Hyalinization & & 0 & 4 & 5 & 1 & 2 \\
\hline Fibrinoid degene & & 0 & 0 & 1 & 0 & 0 \\
\hline Inflammatory ce & ltration & 0 & 1 & 0 & 0 & 0 \\
\hline Occluded artery & ickened intima & 0 & 0 & 0 & 0 & 1 \\
\hline \multicolumn{7}{|c|}{ Retina } \\
\hline Hyalinization & & 0 & 6 & 6 & 0 & 2 \\
\hline
\end{tabular}

*: One dead animal was omitted from the histopathologic evaluation, since the postmortem changes were severe. 

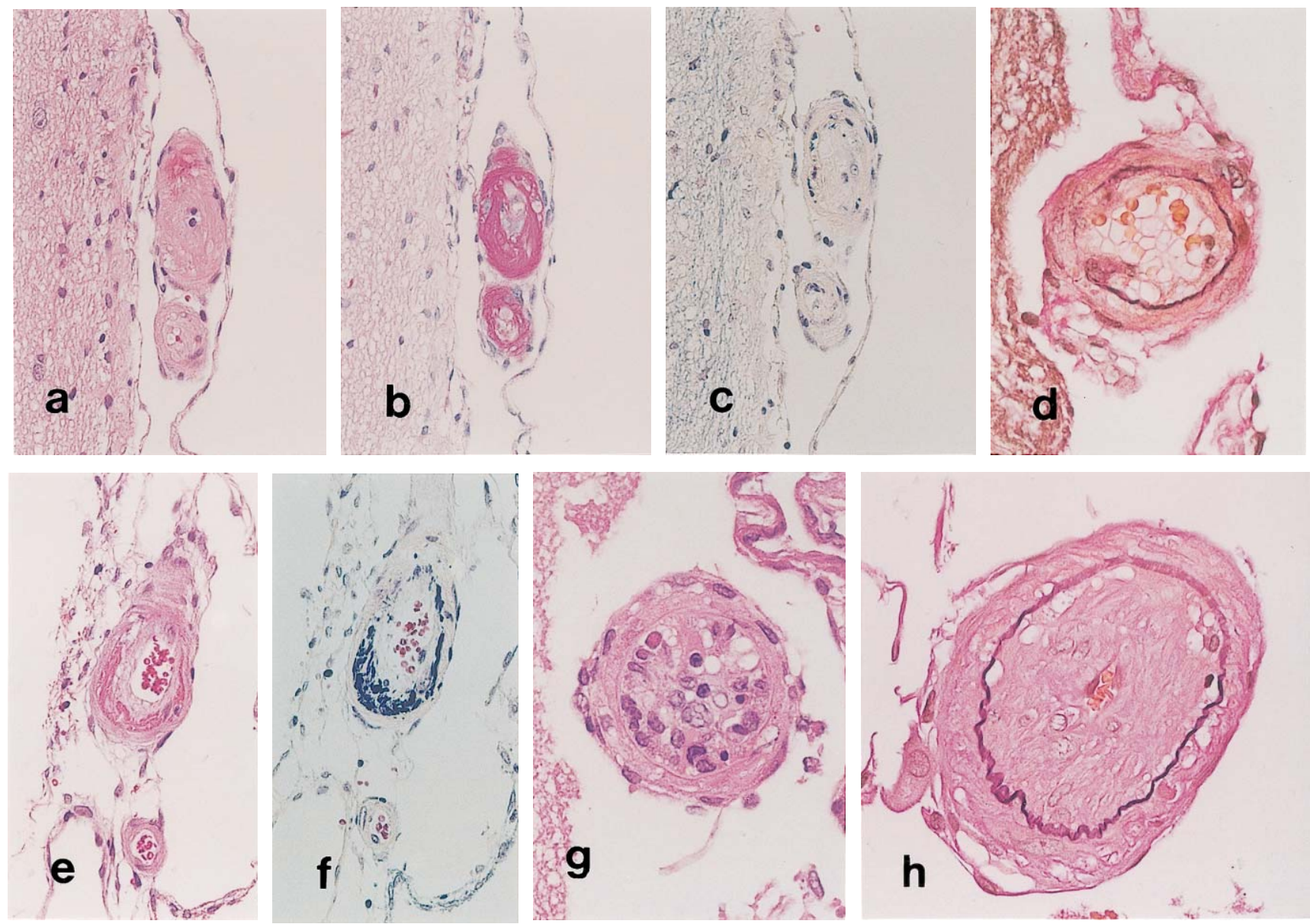

Fig. 2. Photomicrographs of the vascular lesions in the pial artery of the central nervous system from rats receiving $125 \mathrm{mg} / \mathrm{kg}$ per day of IDPN. (a)-(c) Arterial hyalinization in the brain, hyalinized arterial wall is positive for PAS and negative for PTAH, day 44: (a) HE $\times 200$; (b) PAS $\times 200$; (c) PTAH $\times 200$. (d) The internal elastic lamina ruptured in the affected pial artery of the brain, day 44: EVG $\times 400$. (e)-(f) Fibrinoid degeneration in the pial arterial wall of the brain, day 39 (moribund animal): (e) HE $\times 200$; (f) PTAH $\times 200$. (g) Endothelial proliferation with small number of inflammatory cells in the pial arterial wall of the brain, day 30: HE $\times 400$. (h) Arterial intimal thickening of the spinal cord, day 42 (moribund sacrifice): $E V G \times 400$.
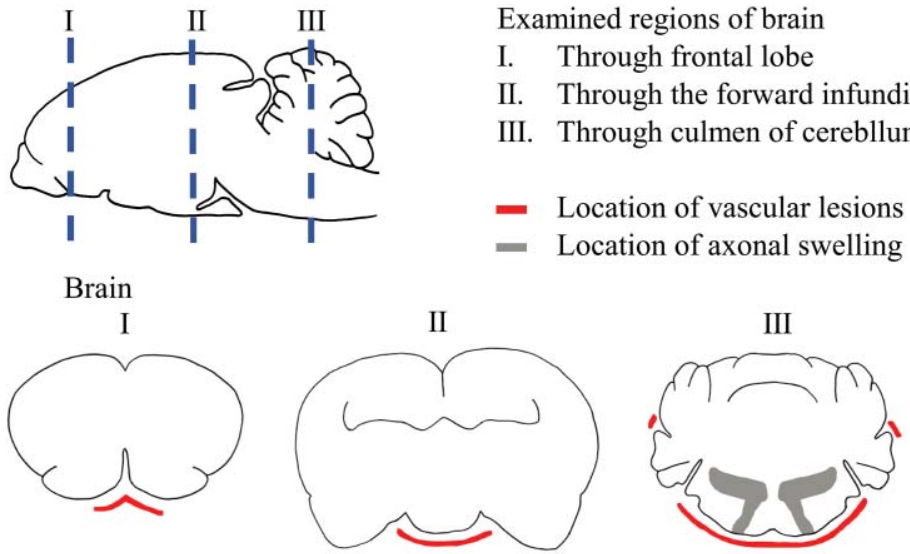

Spinal

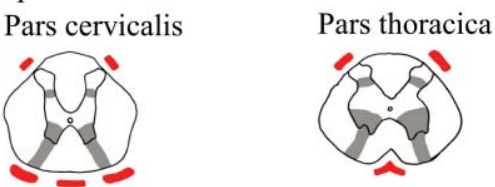

Examined regions of brain

I. Through frontal lobe

II. Through the forward infundibulum

III. Through culmen of cerebllum

- Location of vascular lesions

- Location of axonal swelling 

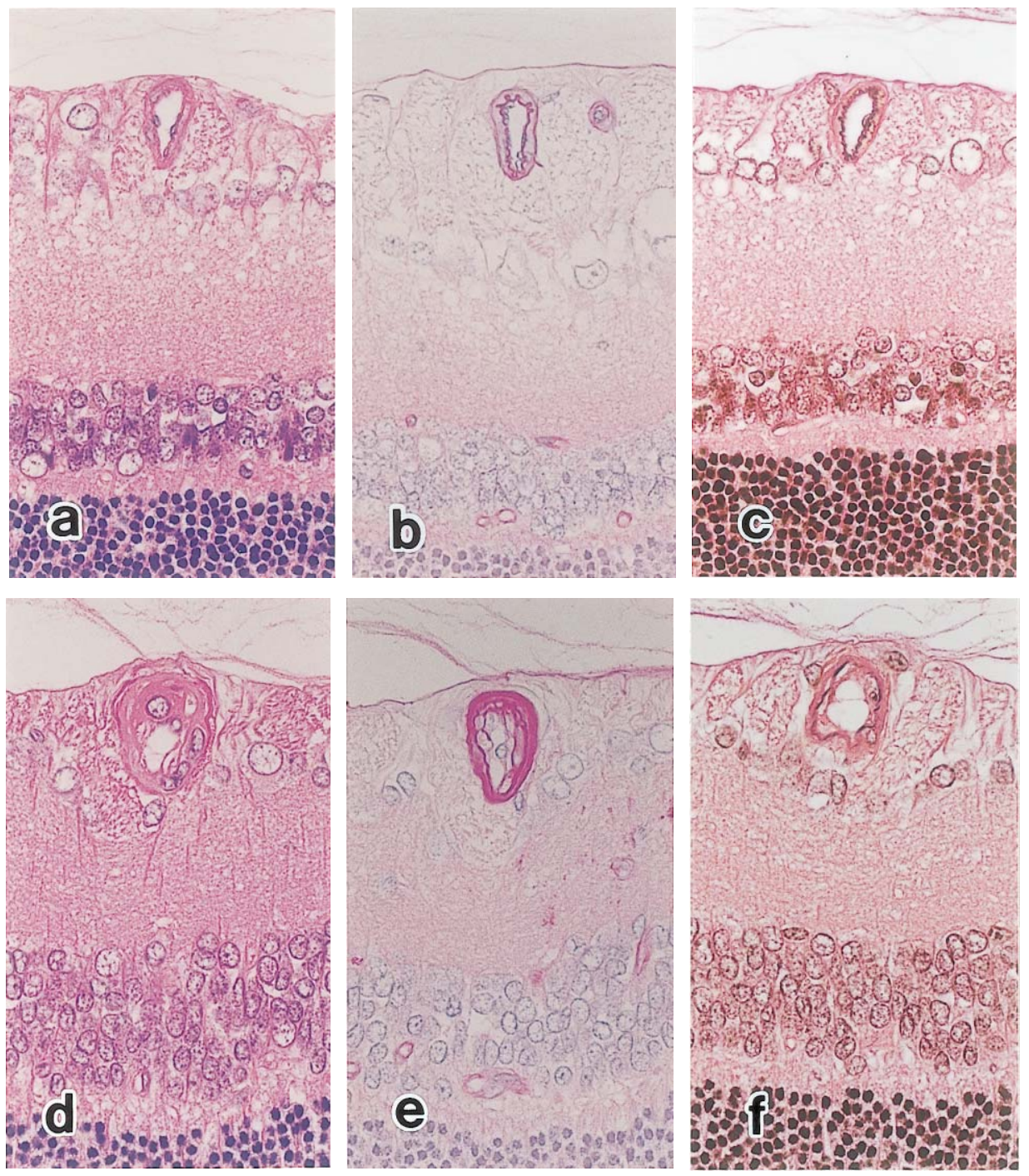

Fig. 4. Photomicrographs of the retina from rats receiving $125 \mathrm{mg} / \mathrm{kg}$ per day of IDPN or purified water, day 44. (a)-(c) No remarkable change is seen in the vessel of the control rat: (a) $\mathrm{HE} \times$ 400; (b) PAS $\times 400$; (c) $E V G \times 400$. (d) - (f) Arterial hyalinization in the IDPN-treated rat: (d) $\mathrm{HE} \times 400$. (e) Deposition of PAS-positive material in the arterial wall: PAS $\times 400$. (f) Disorganized internal elastic lamina: $\mathrm{EVG} \times 400$.

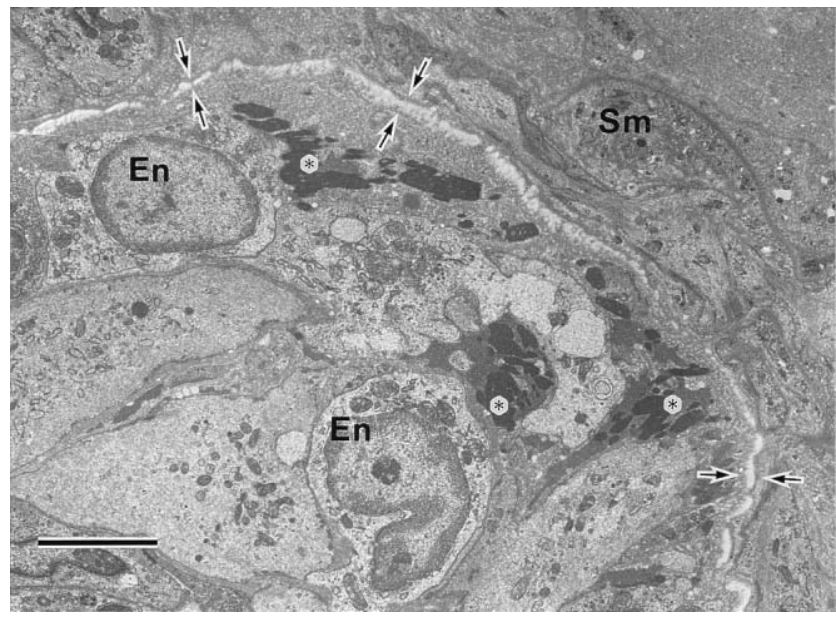

Fig. 5. Electron micrograph of the affected pial artery in the brain from rats receiving $125 \mathrm{mg} / \mathrm{kg}$ per day of IDPN, day 44 . Electron dense amorphous material $(*)$ deposited in the subendothelial space near the internal elastic lamina. Thinning of internal elastic lamina (arrows) is evident. En: Endothelial cell. Sm: Smooth muscle. Bar represents $3 \mu \mathrm{m}$. 


\section{Discussion}

IDPN-induced typical behavioral abnormalities such as circling, head bobbing, and retropulsion which first appeared on day 9 of treatment. Neuropathologic lesion such as proximal axonal swelling was detected on day 15. Arterial hyalinization in the meninx was first detected on day 30 . These results reveal that the IDPN-induced vascular lesion appeared following the behavioral abnormality and the neuropathologic lesion. In the organs and tissues other than the CNS that were examined, vascular lesion was found only in the retina, and the lesions were similar to those observed in the CNS. The lesions in both the CNS and retina were characterized by arterial hyalinization and most of the affected vessels had thickened walls due to the deposition of PAS-positive materials. The retinal arterial hyalinization appeared concomitantly with the vascular lesions of the brain and spinal cord.

The IDPN-induced retinal vascular lesion was investigated for a model of diabetic retinopathy by Heath et al. ${ }^{11,12}$. Microscopic examination using flat preparation demonstrated retinal micro-aneurysms in rats administered a single dose of IDPN. Endothelial proliferation and deposition of PAS-positive material were found in the affected retinal arteries ${ }^{11,12}$. The histopathological features found in the retina and pial artery of the CNS in the present study were similar to these retinal vascular lesions.

According to the results of Heath et al. ${ }^{11,12}$, the collaborative study ${ }^{10}$, and the present study, IDPN appears to be a potent vascular toxicant. The IDPN-induced vascular lesions in the CNS and retina may be caused by the same mechanism, because the lesions in the CNS were similar to those in the retina and their onsets were almost concurrent. However, the mechanism causing the retinal vascular lesions is still unclear. $\beta$-Aminopropionitrile (BAPN), a related non-neurotoxic aminonitrile, is known to induce aneurysm in laboratory animals due to disintegration of the connective tissue induced by the inhibition of lysyl oxidase ${ }^{10,13}$. Similarly in IDPN, a weak inhibitory action of lysyl oxidase has been confirmed ${ }^{13}$. The disintegration of the connective tissue may be a potent factor of IDPN-induced vascular lesions as suggested in the report of the collaborative study ${ }^{10}$.

Our examination of the IDPN-induced vascular lesion by electron microscopy revealed an interesting finding, thinning of the internal elastic lamina. On the assumption that inhibition of lysyl oxidase leads to decreasing rates of collagen and elastin cross-linking ${ }^{10,13}$, the thinning of the internal elastic lamina may be the primary effect of IDPN. However, since our examination by electron microscopy was conducted on one rat only, the thinning of the internal elastic lamina needs to be investigated in depth.

Vascular lesions occur spontaneously in rats and the pancreatic, testicular and mesenteric vessels are most often affected $^{14}$. Various drugs damage the blood vessels and the vulnerable sites vary among drugs. For example, a number of cardiovascular drugs often induce vascular lesions in the mesentery and pancreas of rats. It is well known that phosphodiesterase III inhibitors, vasodilators, induce mesenteric vascular damage in rats ${ }^{14}$. Some anticancer and immunomodulatory drugs often induce vascular lesions in various organs ${ }^{14}$. However, to our knowledge, there have been no previous reports of drug-induced vascular lesions occurring restrictedly in the CNS and retina in an oral dose study using rats. One feature of the IDPN-induced vascular lesions was the characteristic distribution pattern. Since the IDPN-induced vascular lesions occurred after axonal swelling of parenchymal cells around their distribution area, we consider that the occurrence of vascular lesions might be related to neural lesions.

In conclusion, the reproducibility of IDPN-induced vascular lesions in the meninx was confirmed. We showed that IDPN can induce vascular lesions characterized by arterial hyalinization occurring in and restricted to the pia matter of the brain and spinal cord, and retina of the eyes, and that the onset of the lesions is after that of neurotoxicity. In the CNS, almost all of the lesions were found among small sized arteries in the basilar area of the brain and spinal cord, and near the dorsal roots of the spinal cord.

Acknowledgments: The authors wish to thank Ms. Tomoe Asano for excellent assistance in the animal experiment. We also thank Ms. Kanae Tamatsukuri and Mr. Steve Yamakami for language editing.

\section{References}

1. Llorens J, Dememes D, and Sans A. The behavioral syndrome caused by 3,3 '-iminodipropionitrile and related nitriles in the rat is associated with degeneration of the vestibular sensory hair cells. Toxicol Appl Pharmacol. 123: 199-210. 1993.

2. Selye H. Lathyrism. Rev Can Biol. 16: 1-82. 1957.

3. Clark AW, Griffin JW, and Price DL. The axonal pathology in chronic IDPN intoxication. J Neuropathol Exp Neurol. 39: 42-55. 1980.

4. Schulze GE and Boysen BG. A neurotoxicity screening battery for use in safety evaluation: effects of acrylamide and 3,3'-iminodipropionitrile. Fundam Appl Toxicol. 16: 602-615. 1991.

5. Verity MA. Toxic disorders, Experimental neurotoxicity, $\beta$, $\beta$ - iminodipropionitrile. In: Greenfield's Neuropathology, 6th ed., Vol. 1. DI Graham and PL Lantos (eds). Arnold, London. 797. 1997.

6. Genter MB, Llorens J, O'Callaghan JP, Morgan KT, Peele $\mathrm{DB}$, and Crofton KM. Olfactory toxicity of $\beta, \beta$ ' iminodipropionitrile in the rat. J Pharmacol Exp Ther. 263: 1432-1439. 1992.

7. Crofton KM, Janssen R, Prazma J, Pulver S, and Barone S, Jr. The ototoxicity of 3,3'-iminodipropionitrile: functional and morphological evidence of cochlear damage. Hear Res. 80: 129-140. 1994.

8. Llorens J and Dememes D. Hair cell degeneration resulting from 3,3'-iminodipropionitrile toxicity in the rat vestibular epithelia. Hear Res. 76: 78-86. 1994.

9. Seoane A, Espejo M, Pallas M, Rodriguez-Farre E, Ambrosio S, and Llorens J. Degeneration and gliosis in rat 
retina and central nervous system following 3,3'iminodipropionitrile exposure. Brain Res. 833: 258-271. 1999.

10. Yoshioka T, Hamamura M, Yoshimura S, Okazaki Y, Yamaguchi Y, Sunaga M, Hoshuyama S, Iwata H, Okada M, Takei Y, Yamaguchi M, Mitsumori K, Imai K, Narama I, and Okuno Y. Neuropathological evaluation of acrylamideand 3,3'-iminodipropionitrile-induced neurotoxicity in a rat 28-day oral toxicity study: collaborative project for standardization of test procedures and evaluation of neurotoxicity. J Toxicol Pathol. 14: 279-287. 2001.

11. Heath H and Rutter AC. Retinal angiopathy in the imino- dipropionitrile-treated alloxan-diabetic rat. Br J Exp Pathol. 47: 116-120. 1965.

12. Heath H, Paterson RA, and Hart JCD. Changes in the hydroxyproline, hexosamine and sialic acid of the diabetic human and $\beta, \beta$-iminodipropionitrile-treated rat retinal vascular systems. Diabetologia. 3: 515-518. 1967.

13. Wilmarth KR and Froines JR. In vitro and in vivo inhibition of lysyl oxidase by aminopropionitriles. J Toxicol Environ Health. 37: 411-423. 1992.

14. Greaves P. Cardiovascular System, Systemic blood vessels. In: Histopathology of Preclinical Toxicity, 2nd ed. Elserier, London. 278-292. 2000. 